Relations industrielles

Industrial Relations

\title{
Caractères du lock-out
}

\section{Pierre Verge}

Volume 23, numéro 3, 1968

URI : https://id.erudit.org/iderudit/027932ar

DOI : https://doi.org/10.7202/027932ar

Aller au sommaire du numéro

\section{Éditeur(s)}

Département des relations industrielles de l'Université Laval

ISSN

0034-379X (imprimé)

1703-8138 (numérique)

Découvrir la revue

Citer cet article

Verge, P. (1968). Caractères du lock-out. Relations industrielles / Industrial Relations, 23(3), 513-516. https://doi.org/10.7202/027932ar
Résumé de l'article

Un lock-out ne saurait à lui seul servir de fondement à une plainte pour congédiement à la suite d'activité syndicale. Ainsi en décide la C.R.T., après une analyse de ce geste de l'employeur ${ }^{1}$.

(1) Cauchon c. J. D. Chevrolet Oldsmobile Ltée, décision du 26 février 1968, rapportée à (1968) R.D.T. 183.
Tous droits réservés (C) Département des relations industrielles de l'Université Laval, 1968
Ce document est protégé par la loi sur le droit d'auteur. L'utilisation des services d'Érudit (y compris la reproduction) est assujettie à sa politique d'utilisation que vous pouvez consulter en ligne.

https://apropos.erudit.org/fr/usagers/politique-dutilisation/ 
La seconde règle négligée par la Commission, est celle de la conformité au droit naturel. Si l'on ne peut trouver dans le droit naturel, une source directe de droit positif, il est indéniable que le législateur est toujours présumé le respecter. C'est pourquoi, en cas d'ambiguité, l'interprétation conforme à cet ordre de justice naturelle, telle que perçue par les juges, doit être préférée. Les juges Pratte, Casey et Taschereau de la cour d'Appel, en s'appuyant sur des dicta des juges Kerwin et Rand de la cour Suprême, ont donné, dans l'affaire Chabot, une application claire et définitive à cette règle, en interprétant des dispositions législatives et réglementaires, de manière à leur donner un effet conforme à ce qu'ils concevaient être un droit naturel ${ }^{29}$.

A la lumière de ces deux règles d'interprétation, le fondement de la décision apparaît fort fragile sur le plan juridique. L'attitude de la Commission étonne d'autant plus qu'une liberté fondamentale est manifestement en cause!

\section{Caractères du lock-out}

\section{PierRe Verge}

Un lock-out ne saurait d lui seul servir de fondement d une plainte pour congédiement à la suite d'activité syndicale. Ainsi en décide la C.R.T., après une analyse de ce geste de l'employeur ${ }^{1}$.

Le pouvoir accordé à la C.R.T. d'ordonner la réintégration et l'indemnisation d'un salarié dans le cours de son rôle de protection de l'activité syndicale ne saurait jouer que dans le cas où le salarié s'est vu * congédié, suspendu ou déplacé par l'employeur... ${ }^{2}$ » Or, le lockout se distingue, dans ses effets, de chacune de ces situations.

\section{LES FAITS}

Un syndicat accrédité de vendeurs d'automobiles négocie avec une association de marchands un projet de convention collective. La conciliation ne parvient pas à assurer le succès des pourparlers. Aussi, en vient-il à envisager de recourir à la grève, ce qu'il peut alors faire légalement, compte tenu du temps écoulé depuis l'envoi de l'avis de négociation.

(29) Op. cit., pp. 718,722 et 730 .

(1) Cauchon c. J. D. Chevrolet Oldsmobile Ltée, décision du 26 février 1968, rapportée à (1968) R.D.T. 183.

(2) C.t., art. 14 . 
Les employeurs prennent les devants: avant mème que n'ait lieu une assemblée syndicale projetée, au cours de laquelle devait avoir lieu un vote de grève, chacun d'entre eux convoque ses vendeurs pour leur faire part de leur décision commune de recourir au lock-out. La cessation du travail qui s'ensuit amène un salarié à soumettre à la C.R.T. une plainte à l'effet qu'il a été congédié pour activité syndicale, prétention, avons-nous vu, que celle-ci rejette.

Le plaignant, énonce-t-elle, a bien établi qu'il était "salarié ", au sens du Code ${ }^{3}$, qu'il avait exercé "un droit résultant du Code " (en l'occurrence, être membre d'un syndicat ${ }^{4}$ ); il n'a, par contre, pas réussi à établir le dernier élément, à savoir qu'il aurait été " congédié, suspendu ou déplacé ". C'est ici qu'une juste perception de la nature du lock-out devient fatale à la plainte.

\section{CONSIDÉRATIONS SUR LA NATURE DU LOCK-OUT}

A la suite de la C.R.T., constatons que l'on ne cesse pas d'être "salarié " par suite d'un lock-out, ce qui suffit à distinguer ce dernier d’un congédiement (A). Surtout, situons le lock-out, dans le contexte des rapports collectifs du travail (B). On ne saurait ainsi lui assimiler la suspension individuelle d'un salarié.

\section{A. Effet suspensif}

Le Code du travail dispose: "Personne ne cesse d'être un salarié pour l'unique raison qu'il a cessé de travailler par suite de grève ou lock-out " ${ }^{5}$. Malgré le lock-out (légal ou illégal - le Code ne distingue pas), donc, le salarié, temporairement privé de travail, n'en continue pas moins, juridiquement, de se rattacher à l'entreprise qui l'emploie.

Ceci suffit, à distinguer le lock-out du congédiement, ainsi que le reconnaît la C.R.T. dans l'espèce.

Le maintien statutaire du statut de salarié pendant le lock-out (de même que pendant une grève) a pour effet, notamment, que l'individu privé momentanément de travail continue alors de jouir de la protection

(3) Voir la décision Syndicat des vendeurs d'automobiles du district de Québec, (C.S.N.) c. Giguère Automobile Inc., (1967) R.D.T. 321, commentée à (1968) 23 Relations industrielles 165.

(4) La Commission aurait-elle changé d'avis depuis la décision Bergeron c. La Cie d'assurance Les Provinces-Unies? Cette décision est rapportée à (1967) R.D.T. 535 .

(5) C.t., art. 98. La disposition correspondante de la loi fédérale se lit : « Personne ne cesse d'être un employé pour l'unique raison qu'il a cessé de travailler par suite de lock-out ou de grève ou pour l'unique raison de son renvoi contrairement à la présente loi ». (Loi sur les relations industrielles et sur les enquêtes visant les différends du travail, S.R.C., 1952, ch. 152, art. 2 (2).) 
qu'offre le Code du travail relativement à son droit d'association, dans les cas qui y donnent ouverture.

Peut-on dire pour autant que le législateur québécois s'est ainsi trouvé à faire sienne la thèse de la suspension du contrat (individuel) de travail pendant le lock-out (ou la grève)? Le statut, il est vrai, ne contient aucune référence directe au contrat individuel ${ }^{6}$. La notion statutaire de salarié comporte toutefois tous les éléments constitutifs du contrat individuel: prestation de travail, rémunération et subordination.

Retenir, selon le vocabulaire civiliste, la théorie de la suspension du contrat individuel par le lock-out (ou la grève) revient à dire que ce dernier ne rompt pas le lien contractuel entre l'employeur et chacun de ses salariés. Il y a seulement interruption dans l'exécution réciproque des prestations découlant du contrat: fourniture et exécution du travail; paiement du salaire. Conséquence théorique, alors: à la fin du lock-out (ou de la grève), tous les salariés doivent avoir l'occasion de reprendre le travail. Sinon, l'employeur s'expose, de la part de ceux à qui il ne permettrait pas de reprendre le travail, à une poursuite en dommages-intérêts, faute par lui de fournir le travail, comme il s'y était engagé, dans le cas (rare) d'un engagement à durée déterminée, ou encore d'avoir donné le préavis requis, dans le cas (fréquent) d'un engagement à durée indéterminée, à moins, bien entendu, que, dans un cas comme dans l'autre, le salarié n'ait pris sur lui-même de rompre le contrat en s'embauchant ailleurs.

Mais, encore une fois, le Code du travail ne traite expressément Gue du statut de * salarié * et non du contrat individuel comme tel. Essentiellement, il situe le lock-out (et la grève) dans le contexte des rapports collectifs de travail.

\section{B. Aspect collectif}

Le Code du travail présente, en effet, le lock-out comme un procédé de la négociation collective: "le refus par un employeur de fournir du travail à un groupe de salariés à son emploi en vue de les contraindre à accepter certaines conditions de travail... ${ }^{7}{ }^{\star}$ Il ne peut être licite, en ce qui a trait au moment où l'on y a recours, que de façon concomitante à la grève ${ }^{8}$. Grève et lock-out: il y a là a réciprocité de moyen de pression ${ }^{9}$ entre l'employeur et le représentant collectif de ses salariés, le syndicat accrédité ou volontairement reconnu. Ainsi envisagé en tant que procédé de négociation collective, il devient clair que le lock-out atteint globalement un ensemble de salariés, et non, directe-

(6) Il en est autrement en droit français : "La grève ne rompt pas le contrat de travail, sauf faute lourde imputable au salarié ». (Loi du 11 février 1950, art. 4.)

(7) C.t., art. 1, i).

(8) C.t., art. 97.

(9) Sinay, H., La grève, Dalloz, 1966, p. 345. (Le titre VIII de l'ouvrage est consacré à l'étude du lock-out dans le contexte du droit français.) 
ment, chacun de ces derniers. Il se situe au même palier que la grève.

Le lock-out se distingue ainsi du geste par lequel l'employeur impose (pour une raison disciplinaire ou une autre) une suspension à l'un ou quelques-uns de ses salariés.

De façon un peu paradoxale, le fait de situer ainsi le lock-out dans l'ordre des rapports collectifs peut servir à justifier le recours à la théorie de la suspension dans le cas des contrats individuels.

S'il fallait, en effet, s'en tenir au seul engagement de l'employeur à l'endroit de chacun de ses salariés, force serait de conclure à un manquement de sa part, au moment où il a recours au lock-out: il ne fournit pas le travail, comme il l'avait convenu pour un temps plus ou moins long. Pourtant, le Code du travail, voulant assurer le jeu de la négociation collective, permet exceptionnellement, le lock-out. Comment l'employeur pourrait-il véritablement alors exercer ce droit que le statut lui reconnaît, s'il devait être par ailleurs dans l'illégalité compte tenu des engagements qui le lient à ses employés? 10

(Il en va ainsi, plus aisément, dans le cas de la grève. Dans le seul contexte du contrat individuel, l'employé fait défaut d'exécuter le travail comme il l'avait convenu, ce qui justifierait l'employeur de s'en tenir à la rupture du contrat. Comment, alors, pourrait-on sans illusion parler du droit de recourir à la grève? Au surplus, la grève est un geste collectif; on ne peut la réduire à la somme des refus individuels de travailler. Ceci vaut d'ailleurs que la grève soit légale ou illégale: s'il y a illégalité, elle est collective, non individuelle ${ }^{11}$.)

Le Code du travail détermine la légalité du lock-out dans le contexte des rapports collectifs, ne permettant d'y recourir que lorsque le syndicat a de son côté acquis le droit de faire grève. Le monopole de la représentation syndicale, dont jouit alors le syndicat, a donc paru rétablir l'équilibre des forces lors de la détermination collective des conditions de travail.

Le législateur n'ayant pas par ailleurs jugé bon d'intégrer dans cette législation le champ des rapports individuels de travail (dans la mesure où ceux-ci peuvent encore avoir une signification réelle), l'on en est réduit à tenter de concilier deux âges.

(10) En droit français, le professeur Brun était d'avis que pour juger de la validité du lock-out, il fallait «... l'examiner non point en se plaçant sur le terrain des relations individuelles, mais dans le cadre des seuls rapports collectifs $\gg$. (Brun, A., et Galland, H., Droit du travail, Sirey, 1958, p. 923). Il devait constater, quelques années plus tard, que la jurisprudence française, envisageant le lock-out avant tout en rapport avec les obligations découlant du contrat individuel, "(avait) évolué dans un sens nettement restrictif du droit de lock-out ». (Mise à jour, 1962, p. 185. Voir aussi, Sinay, op. cit., pp. 350 et suivantes.)

(11) Me M.-L. BEAUlieu, Les conflits de droit dans les rapports collectifs du travail, Presses universitaires Laval, 1955, p. 461. 